\title{
Pelvic venous insufficiency - an often-forgotten cause of chronic pelvic pain
}

\author{
Jacek Szymanski@, Grzegorz Jakiel@, Aneta Slabuszewska-Jozwiak@ \\ ${ }^{15 t}$ Department of Obstetrics and Gynecology, Centre of Postgraduate Medical Education, Warsaw, Poland
}

\begin{abstract}
Chronic pelvic pain is a common health problem that afflicts $39 \%$ of women at some time in their life. It is a common challenge for gynecologists, internists, surgeons, gastroenterologists, and pain management physicians. Pelvic venous insufficiency (PVI) accounts for $16-31 \%$ of cases of chronic pain but it seems to be often overlooked in differential diagnosis. The aim of this article was to summarize current data concerning PVI. The embolization of insufficient ovarian veins remains the gold standard of therapy but the optimal procedure is yet to be determined. Well-designed randomized trials are required to establish the best treatment modalities.
\end{abstract}

Key words: chronic pelvic pain; pelvic venous insufficiency; pelvic congestion syndrome; embolization

Ginekologia Polska 2020; 91, 11: 704-708

The term chronic pelvic pain (CPP) refers to a pain syndrome experienced by women that lasts more than six months and negatively impacts their everyday activities to a high degree, decreasing their quality of life. The pain is hardly associated with the menstrual cycle and pregnancy but can be exacerbated by some hormonal or behavioral conditions (sexual intercourse). The origin of this bothersome syndrome is related to a variety of pathologies. CPP afflicts millions of women worldwide and often requires a multi-specialization medical approach in the diagnostic and therapeutic process. The incidence of CPP in the UK is estimated to be $38 / 1,0000$ patients per year [1]. Since the diagnosis of chronic pelvic pain still remains a challenge, one third of the patients who are evaluated yield no clear etiology. Subsequently, one third of those with no apparent cause of pelvic pain have pelvic venous insufficiency (PVI). Two definitions related to this condition are often mixed and covered by the term CPP. Pelvic (venous) congestion syndrome (PCS) refers to chronic pelvic pain resulting from pelvic venous distention. However, due to the lack of detailed diagnostic criteria, PCS should not be a recognized entity. Some data suggest that CPP could be secondary to PVI, with clinical manifestations of pelvic and vulvar discomfort, dyspareunia as well as lower back pain. The symptoms usually potentiate in the evening and often release in a supine position [2]. The pathophysiology of $\mathrm{PVI}$ is related to retrograde flow through incompetent gonadal and pelvic veins. This pathology can result from primary vulvar insufficiency, venous outflow obstruction, and hormonally mediated vasomotor dysfunction. The term PVI should be preferred as it seems to be the closest to the pathological background of this condition [3].

The problem affects women of childbearing age, suggesting its relation to hormonal status. Animal studies have shown ovarian vain distention when exposed to increased doses of estrogens. Furthermore, the exacerbation of signs and symptoms is demonstrated during pregnancy and the menstrual cycle with relief from pain occurring after the menopause [4]. Multiparity seems to be a risk factor for PVI. Vein capacity during pregnancy can increase by as much as $60 \%$. Additionally, the anatomical changes in the pelvis and increase in weight during pregnancy may result in temporary venous obstruction [5]. From a mechanical point of view, venous dilatation could result from incompetent valves and retrograde venous flow. The venous system of the pelvis builds an anastomotic plexus with the main direction of the blood flow going from the uterus, parametrium, and mesosalpinx to the internal iliac and ovarian veins [6]. In typical anatomic conditions, the left ovarian vein drains to the left renal vein and the vena cava receives the right ovarian vein. Anatomical variabilities may cause an obstruction of the blood flow leading to venal dilatation and insufficiency. Additionally, compression of the left ovarian vein by the superior mesenteric artery may occur (nutcracker phenom- 
enon) and the left iliac vein may be compressed against the lumbar vertebral bodies by the right common iliac artery, which could result in endothelial damage, thickening, and venous congestion (May-Thurner syndrome) [5, 7]. Imaging studies have shown that an ovarian vein diameter greater than $8 \mathrm{~mm}$ is associated with significantly wider peri-uterine veins compared to a diameter less than $8 \mathrm{~mm}$ [3]. In general, two physiopathological mechanisms underlie this condition:

1. Venous outflow obstruction impeding the centripetal direction of flow.

2. Venous vulvar dysfunction and venous leakage through collateral routes, permitting flow reentry in a centrifugal (proximal-to-distal) direction [8].

One of the classifications of pelvic varicose veins distinguishes three types of vein damage:

1. Vascular wall pathology (valvular incompetence, malformations or agenesis);

2. Vascular compression effects such as the nutcracker syndrome or the May-Thurner syndrome; and

3. Pathology-induced (e.g., endometriosis or pelvic tumor) local extrinsic compression [6].

Pelvic congestion syndrome is usually related to incompetent internal pudendal and broad ligament parametrial branches, whilst vulvar and lower limb varicosities refer to incompetent branches of the circumflex femoral and obturator veins [9].

\section{CLINICAL PRESENTATION}

Most women suffer from prolonged noncyclical pelvic pain accompanied by dyspareunia and post-coital discomfort, bladder irritation, and dysmenorrhea. The pain may increase while sitting, standing, at the end of the day, or just before the onset of the menses. Additionally, vaginal discharge, vulvar edema, and tenderness of the pelvis that may contribute to depression could also be present. Vulvar varicosities and varicose veins on the posterior surfaces of the thighs may be found in physical examination. A gynecological examination often reveals uterine dyskinesia and palpatory tenderness over the ovaries. Moreover, varices of the vulva, vagina, and in perianal area are often identified. One in five women presenting with varicose veins have reflux of non-saphenous origin, with the associated pelvic vein reflux present in one in six [10]. The differential diagnosis should include endometriosis, pelvic inflammatory disease (PID), ovarian tumors, bowel pathology, fibroids, pelvic organ prolapse, urologic pathology, and porphyria $[5,6]$. A case of PCS initially misdiagnosed as a hydrosalpinx was also reported [11].

\section{DIAGNOSIS}

Pelvic ultrasound (US) is typically the first-line imaging modality in the diagnostic process of patients with chronic pelvic pain. The advantage of a Doppler ultrasound examination is the ability to provide information about venous blood flow. Beard et al. have established criteria for the sonographic diagnosis of pelvic varices:

1. Visualization of the ovarian veins dilated above $4 \mathrm{~mm}$ in diameter,

2. Communication of the dilated tortuous arcuate veins in the myometrium with bilateral pelvic varicose veins,

3. Blood flow less than $3 \mathrm{~cm} / \mathrm{s}$, and

4. Detection of retrograde venous blood flow in the left ovarian vein [12].

The dilatation of pelvic veins $>8 \mathrm{~mm}$ is associated with reflux and symptoms, while the diameter $4-8 \mathrm{~mm}$ is usually related to asymptomatic reflux [13]. In contrary, Dos Santos et al. showed no significant difference between the diameters of competent and refluxing ovarian veins, concluding that it is not acceptable to use vein diameter as an indicator of ovarian venous reflux [14]. Park et al. [15], with a combination of transabdominal and transvaginal Doppler ultrasound in the evaluation of women with CPP and PVI, found that the mean left ovarian vein diameter was greater in symptomatic patients as compared to healthy controls $(0.79$ vs $0.49 \mathrm{~cm}, \mathrm{p}<0.001)$. Furthermore, the authors reported a sensitivity of $100 \%$ and specificity of $75 \%$ when evaluating retrograde flow in gonadal veins with the use of transabdominal ultrasound. In another study, the sensitivity and specificity of duplex ultrasound for the detection of a dilated left ovarian vein (LOV) were $100 \%$ and $57 \%$, and for the right ovarian vein (ROV) they were $67 \%$ and $90 \%$ [16]. The high efficiency of US in detecting venal reflux was confirmed in the study by Hansrani et al. [17]. The authors reported better visibility of pelvic veins in the supine position against the semi-standing position (76\% vs $64 \%$ ), but better identification of pelvic vein incompetence in the semi-standing position as compared to the supine position ( $76 \%$ vs $68 \%$ ), concluding that a complete US evaluation should be performed in both cases, including the Valsalva maneuver.

Magnetic resonance imaging (MRI) provides excellent pelvic organ imaging and can be utilized for PVI detection. Although, it carries the risk of underestimation of the extent of the pelvic venous plexus and dilatation of the gonadal vein, when performed in dorsal decubitus, several studies showed its high sensitivity and specificity reaching $88-100 \%$ and $38-75 \%$, respectively $[2,18]$. A comparable efficacy in the assessment of flow velocity and reflux in ovarian veins is observed in phase-contrast MRI. However, the time-resolved MRI achieved the best efficacy in the imaging of retrograde flow in ovarian veins, with an accuracy of $79-85 \%$ as compared to conventional venography. Time-resolved imaging (TRI) is a specialized 3D contrast-enhanced MR angiographic sequence particularly useful for venous imaging and indi- 
cated in cases when a proper diagnosis is dependent on the presence and direction of the flow. TRI is effective in the detection of non-dilated incompetent ovarian veins and dilated but competent ovarian veins and, contrary to conventional venography, reveals possible arteriovenous (AV) malformations [19]. Conventional venography is an invasive procedure but still constitutes the criterion standard for the evaluation of PVI. The diagnostic criteria for PVI include:

1. Dilatation of the uterine and ovarian veins above $5 \mathrm{~mm}$,

2. Presence of retrograde flow in gonadal veins,

3. Stasis of contrast material in pelvic venous plexus, and

4. Opacification of vulvar and thigh varices.

5. Retrograde flow of contrast material through the utero-ovarian arcade to the opposite side [3].

In 2019, a consensus was achieved among UK-based interventional radiologists on the optimal imaging strategy and definition of important imaging diagnostic features in women with PVI. Three consensus statements were defined:

1. Catheter venography is the gold standard investigation for the diagnosis of pelvic vein incompetence;

2. PVI should be defined as "retrograde flow along the ovarian or internal iliac veins"

3. Pelvic varices should be defined as "tortuous, often dilated, vulval, adnexal, para-uterine veins arising from incompetent internal iliac or ovarian veins" [20]. Anyhow, it seems that noninvasive imaging modalities are efficient enough to make a proper diagnosis of PVI. In summary, the most important imaging feature for the diagnosis of PVI is the demonstration of a reflux in one or both gonadal veins. Furthermore, the presence of a dilated vain which crosses the midline of the uterus seems to be the most specific sign of PVI [18].

The differential diagnosis of CPP could be long and trying as chronic pain is often the result of a multiple, overlapping pain condition, with each contributing to the generation of pain. Other gynecologic causes of pain in the pelvis should include endometriosis with adenomyosis, intra-abdominal adhesions, leyomyomas, ovarian remnant and residual ovary syndrome, gynecologic malignancies, vulvodynia, and dyspareunia. Endometriosis is the most common gynecologic cause of CPP and the coexistence of other pain syndromes in women with endometriosis is higher than in the general population [21]. However, the presence and severity of endometriosis does not often correlate with symptom severity. Adenomyosis coexists in approximately $20 \%$ of women with deeply infiltrative endometriosis, however, the relationship between adenomyosis and CPP is not fully understood [22]. As it had been shown in the Swann study, adenomyosis is frequently identified in asymptomatic women with no correlation to pelvic pain or abnormal bleeding [23]. The relationship between CPP and abdominal adhesions is poorly defined, however, there is some evidence that dense adhesions limiting organ mobility may cause pelvic pain [24]. Uterine leiomyomas are hardly related to chronic pain, but in one survey CPP was reported by $15 \%$ of women with fibroids as compared to $3 \%$ by women without fibroids [25]. Ovarian remnant syndrome refers to patients who have undergone bilateral oophorectomy with ovarian tissue inadvertently left behind, while residual ovarian syndrome is related to patients who had ovarian conservation and subsequently developed pathology. These patients may present cyclic or chronic pain with acute flare-ups [26]. Chronic pelvic pain is usually a complex condition, thus, the differential diagnosis should consider the signs and symptoms of gastrointestinal tract dysfunction such as: irritable bowel syndrome, inflammatory bowel disease, diverticular colitis, celiac disease; urinary tract dysfunction like painful bladder syndrome and recurrent urinary tract infections; neurologic causes as nerve injury or central sensitization, and musculoskeletal causes, for instance, myofascial pelvic pain syndromes or fibromyalgia. Depression and other psychiatric comorbidities, opioid dependency, and sexual abuse in the history should also be included into clinical investigation.

\section{TREATMENT}

A conservative medical management is suggested by several authors as a first-line therapy. The data are limited as they come from small randomized trials. Women treated with goserelin (3.6 mg per month), medroxyprogesterone acetate (30-50 mg per day) or an etonogestrel implant reported improved pain and venography scores [27-29].

Patients who do not respond to medical therapy can pursue invasive treatment. Embolization is the gold standard in the treatment of PVI. Insufficient ovarian veins generate a hydrostatic pressure that by embolization could be eliminated. Embolization is usually performed on an outpatient basis. The procedure of embolization of bilateral ovarian veins in PCS treatment was introduced by Edwards in 1993 [30]. Usually the transfemoral or transjugular approach is used to achieve gonadal and internal iliac pelvic vein access. Transcatheter embolization is performed after gonadal venous insufficiency is confirmed by venography. For clinical success, scrupulous and complete embolization is crucial as there are multiple tributaries of the ovarian veins and many collateral veins supporting the flow between the contralateral sides of the pelvis. For this purpose, embolization should cover the main ovarian veins with their tributaries to the level of the inferior vena cava (IVC) on the right side and a level of $3 \mathrm{~cm}$ from the renal vein on the left. Foamed sclerosants, such as $3 \%$ sodium tetradecyl sulfate or $5 \%$ sodium morrhuate, are often used as adjuncts to coils to reduce recanalization and treatment failure. Platinum coils are MRI compatible up to $1.5 \mathrm{~T}$ and go undetected by 
airport scanners. Usually six coils are sufficient for complete embolization but the number can vary from two to ten depending on the clinical situation [6]. Complete occlusion is obtained by gradually untwisting the fibered platinum coil (FPC) along the vessel [9]. One of the studies showed the high effectiveness of the ethylene vinyl alcohol copolymer as a sclerosant in the embolization of the ovarian vein [31]. Gonadal, vena cava, and internal iliac vein venography should be finally performed to confirm the gonadal vein occlusion with subsequent selective embolization if residual reflux into ovarian, vulvar, or thigh region varices is present [3].

The initial venous puncture and embolization process should be performed with reasonable care to avoid serious complications. Intraoperative complications include: hematoma, pneumothorax due to venous puncture, and embolization of non-target vessels (coil misplacement). Stroke caused by an emboli from coil migration or uncontrolled foam has been also reported. Delayed complications include an enlarging pneumothorax and coil migration [9].

PVI coexists with leg varicosities in approximately $76 \%$. Embolization of incompetent pelvic veins seems to be mandatory in cases of lower limb varicose veins. In one study, embolization led to an improvement of PCS in $91 \%$, and of lower limb varicose veins in $51 \%$ on its own [32]. Furthermore, there is high incidence of $\mathrm{PVI}$ in patients with recurrent varices after surgery (REVAS). Monedero et al. [8] reported a significant relief of clinical signs and symptoms of pelvic and lower extremity venous stasis after the embolization of gonadal and hypogastric mainstem and collateral vessels in patients with REVAS and PVI. In contrary, Rabe and Panier, based on a literature review, did not find satisfactory evidence for the efficiency of ovarian and pelvic vein embolization in the treatment of varicose veins of pelvic origin in patients without PCS. The Authors suggest performing foam sclerotherapy or phlebectomy in these patients [33].

Abdelsalam reported a 3-year follow-up of 11 patients with lower abdominal pain and vulvar varicosities treated with unilateral ovarian vein (OV) embolization (6 cases) or bilateral OV embolization (5 cases) with the use of spiral coils. The procedural success rate was $100 \%$. Post-embolization pain relief and relief of vulvar varices were encountered in $70 \%$ of patients within 3 months of the procedure; however, in 1 patient (10\%) the symptoms returned after 6 months. No significant complications were reported [34]. The effectiveness of ovarian vein embolization was confirmed in the study by Pyra, in which the clinical success was reflected by a decrease in the visual analogue scale (VAS) from 8 points at the baseline to 1 point at the 3-month follow-up ( $p<0.001$ ) [35]. Guirola et al. [36] compared the efficacy of vascular plugs (VPs) and FPCs for embolization in PVI. They found no statistically significant differences in clinical success and subjective improvement concerning dyspareunia, dysmenorrhea, urinary urgency, and pelvic pain at 1-year follow-up.VPs were associated with decreased fluoroscopy time and radiation dose but also with a significantly higher cost of therapy.

As revealed by Riding et al. [2], a substantial number of studies reporting significantly improved patient outcomes following endovascular embolization are of a relatively low quality, varying in terms of patient demographics, inclusion criteria, various embolization procedures performed, and different outcome measures used. There is also heterogeneity among the diagnostic criteria for PVI and CPP. The approach to the therapy and treatment modalities varies among vascular specialists. A survey conducted in the UK revealed that $9 \%$ of them do not regard pelvic vein reflux as a pathological entity and $11 \%$ never investigated or treated it. Indications for investigation include labial (94\%) and buttock/upper thigh (70\%) varicose veins where $46 \%$ used MR venography and $16 \%$ transvaginal duplex. The treatment modalities include transcatheter coil embolization (89\%), sclerotherapy via the thigh varicose vein (47\%), and transcatheter sclerotherapy (26\%). Both ovarian veins and internal iliac tributaries were treated by $61 \%$ of responders, while $34 \%$ treated only ovarian vein reflux. Such substantial variation in the management of pelvic vein reflux requires well-designed clinical trials to establish recommendations for good clinical practice [37].

Chronic pelvic pain still remains a diagnostic and therapeutic challenge. Due to its complexity, it often requires a multidisciplinary approach involving gynecologists, vascular surgeons, and interventional radiologists. PVI, as a cause of CPP, seems to be underestimated, although it can be successfully treated when properly diagnosed. The therapeutic uncertainty raises questions about the number of veins that should be closed, the kind of embolization material used, and the treatment of vulval varicosities in patients with asymptomatic PVI. Well-designed, prospective long-term trials are needed to clarify these issues and to establish recommended guidelines for clinicians.

\section{REFERENCES}

1. Zondervan KT, Yudkin PL, Vessey MP, et al. Prevalence and incidence of chronic pelvic pain in primary care: evidence from a national general practice database. Br J Obstet Gynaecol. 1999; 106(11): 1149-1155, doi: 10.1111/j.1471-0528.1999.tb08140.x, indexed in Pubmed: 10549959.

2. Riding DM, Hansrani V, McCollum C. Pelvic vein incompetence: clinical perspectives. Vasc Health Risk Manag. 2017; 13: 439-447, doi: 10.2147/VHRM.S132827, indexed in Pubmed: 29225469.

3. Black CM, Thorpe $K$, Venrbux $A$, et al. Research reporting standards for endovascular treatment of pelvic venous insufficiency. J Vasc Interv Radiol. 2010; 21(6): 796-803, doi: 10.1016/j.jvir.2010.02.017, indexed in Pubmed: 20494288.

4. Stones RW. Pelvic vascular congestion-half a century later. Clin Obstet Gynecol. 2003; 46(4): 831-836, doi: 10.1097/00003081-20031200000013 , indexed in Pubmed: 14595225.

5. Ignacio EA, Dua R, Sarin S, et al. Pelvic congestion syndrome: diagnosis and treatment. Semin Intervent Radiol. 2008; 25(4): 361-368, doi: 10.1055/s-0028-1102998, indexed in Pubmed: 21326577. 
6. Corrêa M, Bianchini L, Saleh J, et al. Síndrome da congestão pélvica e embolização de varizes pélvicas. Jornal Vascular Brasileiro. 2019; 18, doi: 10.1590/1677-5449.190061.

7. Ruel J. May-Thurner Syndrome: An often-missed cause of chronic pelvic pain. J Am Assoc Nurse Pract. 2019; 31(7): 388-390, doi: 10.1097/JXX.0000000000000205, indexed in Pubmed: 30908405.

8. Monedero J, Ezpeleta S, Castro J, et al. Embolization treatment of recurrent varices of pelvic origin. Phlebology: The Journal of Venous Disease. 2016; 21(1): 3-11, doi: 10.1258/026835506775971108.

9. Lopez AJ. Female pelvic vein embolization: indications, techniques, and outcomes. Cardiovasc Intervent Radiol. 2015; 38(4): 806-820, doi: 10.1007/s00270-015-1074-7, indexed in Pubmed: 25804635.

10. Marsh $\mathrm{P}$, Holdstock J, Harrison $C_{\text {, }}$ et al. Pelvic vein reflux in female patients with varicose veins: comparison of incidence between a specialist private vein clinic and the vascular department of a National Health Service District General Hospital. Phlebology. 2009; 24(3): 108-113, doi: 10.1258/phleb.2008.008041, indexed in Pubmed: 19470861.

11. Czuczwar P, Stępniak A, Szkodziak $P$, et al. Pelvic congestion syndrome initially misdiagnosed as a hydrosalpinx - a diagnostic challenge. Ginekol Pol. 2017; 88(3): 166, doi: 10.5603/GP.a2017.0032, indexed in Pubmed: 28397208.

12. Beard RW, Highman JH, Pearce $S$, et al. Diagnosis of pelvic varicosities in women with chronic pelvic pain. Lancet. 1984; 2(8409): 946-949, doi: 10.1016/s0140-6736(84)91165-6, indexed in Pubmed: 6149342.

13. Nascimento AB, Mitchell DG, Holland G. Ovarian veins: magnetic resonance imaging findings in an asymptomatic population. J Magn Reson Imaging. 2002; 15(5): 551-556, doi: 10.1002/jmri.10098, indexed in Pubmed: 11997896.

14. Dos Santos SJ, Holdstock JM, Harrison CC, et al. Ovarian Vein Diameter Cannot Be Used as an Indicator of Ovarian Venous Reflux. Eur J Vasc Endovasc Surg. 2015; 49(1): 90-94, doi: 10.1016/j.ejvs.2014.10.013, indexed in Pubmed: 25457295.

15. Park SJ, Lim JW, Ko YT, et al. Diagnosis of pelvic congestion syndrome using transabdominal and transvaginal sonography. AJR Am J Roentgenol. 2004; 182(3): 683-688, doi: 10.2214/ajr.182.3.1820683, indexed in Pubmed: 14975970.

16. Malgor RD, Adrahtas D, Spentzouris G, et al. The role of duplex ultrasound in the pelvic congestion syndrome workup. JVasc Surg Venous Lymphat Disord. 2013; 1(1): 107-38, doi: 10.1016/j.jvsv.2012.10.027, indexed in Pubmed: 26993924.

17. Hansrani V, Dhorat Z, McCollum CN. Diagnosing of pelvic vein incompetence using minimally invasive ultrasound techniques. Vascular. 2017; 25(3): 253-259, doi: 10.1177/1708538116670499, indexed in Pubmed: 27688293.

18. Bookwalter CA, VanBuren WM, Neisen MJ, et al. Imaging appearance and nonsurgical management of pelvic venous congestion syndrome. Radiographics. 2019; 39(2): 596-608, doi: 10.1148/rg.2019180159, indexed in Pubmed: 30844351.

19. Chennur VS, Nzekwu EV, Bhayana D, et al. MR venography using time-resolved imaging in interventional management of pelvic venous insufficiency. Abdom Radiol (NY). 2019; 44(6): 2301-2307, doi: 10.1007/s00261-019-01965-w, indexed in Pubmed: 30847564.

20. Riding DM, Pond EJ, McCollum C, et al. Seeking consensus amongst UK-based interventional radiologists on the imaging diagnosis of pelvic vein incompetence in women with chronic pelvic pain: A modified Delphi study. Phlebology. 2019; 34(7): 486-495, doi: 10.1177/0268355518821554, indexed in Pubmed: 30621525.

21. Smorgick N, Marsh CA, As-Sanie S, et al. Prevalence of pain syndromes, mood conditions, and asthma in adolescents and young women with endometriosis. J Pediatr Adolesc Gynecol. 2013; 26(3): 171-175, doi: 10.1016/j.jpag.2012.12.006, indexed in Pubmed: 23507008.

22. Di Donato N, Montanari G, Benfenati A, et al. Prevalence of adenomyosis in women undergoing surgery for endometriosis. Eur J Obstet Gynecol
Reprod Biol. 2014; 181: 289-293, doi: 10.1016/j.ejogrb.2014.08.016, indexed in Pubmed: 25201608.

23. Weiss G, Maseelall $P$, Schott $L L$, et al. Adenomyosis a variant, not a disease? Evidence from hysterectomized menopausal women in the Study of Women's Health Across the Nation (SWAN). Fertil Steril. 2009; 91(1): 201-206, doi: 10.1016/j.fertnstert.2007.11.025, indexed in Pubmed: 18243177.

24. Practice Committee of American Society for Reproductive Medicine in collaboration with Society of Reproductive Surgeons. Pathogenesis, consequences, and control of peritoneal adhesions in gynecologic surgery: a committee opinion. Fertil Steril. 2013; 99(6): 1550-1555, doi: 10.1016/j.fertnstert.2013.02.031, indexed in Pubmed: 23472951.

25. Zimmermann A, Bernuit D, Gerlinger C, et al. Prevalence, symptoms and management of uterine fibroids: an international internet-based survey of 21,746 women. BMC Womens Health. $2012 ; 12: 6$, doi: 10.1186/14726874-12-6, indexed in Pubmed: 22448610.

26. Magtibay PM, Nyholm JL, Hernandez $\mathrm{JL}$, et al. Ovarian remnant syndrome. Am J Obstet Gynecol. 2005; 193(6): 2062-2066, doi: 10.1016/j. ajog.2005.07.067, indexed in Pubmed: 16325616.

27. Soysal ME, Soysal S, Vicdan K, et al. A randomized controlled trial of goserelin and medroxyprogesterone acetate in the treatment of pelvic congestion. Hum Reprod. 2001; 16(5): 931-939, doi: 10.1093/humrep/16.5.931, indexed in Pubmed: 11331640.

28. Farquhar $\mathrm{CM}$, Rogers $\mathrm{V}$, Franks $\mathrm{S}$, et al. A randomized controlled trial of medroxyprogesterone acetate and psychotherapy for the treatment of pelvic congestion. Br J Obstet Gynaecol. 1989; 96(10): 1153-1162, doi: 10.1111/j.1471-0528.1989.tb03190.x, indexed in Pubmed: 2531611.

29. Shokeir T, Amr M, Abdelshaheed M. The efficacy of Implanon for the treatment of chronic pelvic pain associated with pelvic congestion: 1-year randomized controlled pilot study. Arch Gynecol Obstet. 2009; 280(3): 437-443, doi: 10.1007/s00404-009-0951-1, indexed in Pubmed: 19190927.

30. Edwards RD, Robertson IR, MacLean AB, et al. Case report: pelvic pain syndrome - successful treatment of a case by ovarian vein embolization. Clin Radiol. 1993; 47(6): 429-431, doi: 10.1016/s0009-9260(05)81067-0, indexed in Pubmed: 8519153.

31. Marcelin C, Izaaryene J, Castelli M, et al. Embolization of ovarian vein for pelvic congestion syndrome with ethylene vinyl alcohol copolymer (Onyx). Diagn Interv Imaging. 2017; 98(12): 843-848, doi: 10.1016/j. diii.2017.05.011, indexed in Pubmed: 28647478.

32. Hartung O. Embolization is essential in the treatment of leg varicosities due to pelvic venous insufficiency. Phlebology. 2015; 30(1 Suppl): 81-85, doi: 10.1177/0268355515569129, indexed in Pubmed: 25729072.

33. Rabe E, Pannier F. Embolization is not essential in the treatment of leg varices due to pelvic venous insufficiency. Phlebology. 2015; 30(1 Suppl): 86-88, doi: 10.1177/0268355515569412, indexed in Pubmed: 25729073.

34. Abdelsalam $\mathrm{H}$. Clinical outcome of ovarian vein embolization in pelvic congestion syndrome. Alexandria Journal of Medicine. 2019; 53(1): 15-20, doi: 10.1016/j.ajme.2016.01.006.

35. Pyra K, Woźniak S, Roman T, et al. Evaluation of effectiveness of endovascular embolisation for the treatment of pelvic congestion syndrome - preliminary study. Ginekol Pol. 2015; 86(5): 346-351, doi: 10.17772/gp/2420, indexed in Pubmed: 26117971.

36. Guirola JA, Sánchez-Ballestin M, Sierre S, et al. A Randomized Trial of Endovascular Embolization Treatment in Pelvic Congestion Syndrome: Fibered Platinum Coils versus Vascular Plugs with 1-Year Clinical Outcomes. J Vasc Interv Radiol. 2018; 29(1): 45-53, doi: 10.1016/j. jvir.2017.09.011, indexed in Pubmed: 29174618.

37. Campbell B, Goodyear S, Franklin I, et al. Investigation and treatment of pelvic vein reflux associated with varicose veins: Current views and practice of 100 UK vascular specialists. Phlebology. 2020; 35(1): 56-61, doi: 10.1177/0268355519848621, indexed in Pubmed: 31084296. 